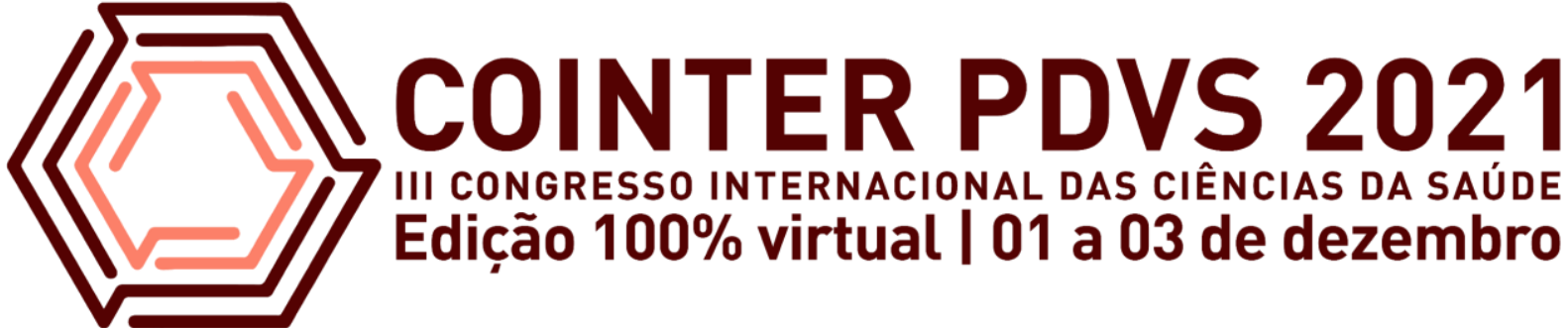

\section{CUIDADOS DE ENFERMAGEM ACERCA DA VENTILAÇÃO NÃO INVASIVA EM PACIENTES COM DOENÇA PULMONAR OBSTRUTIVA CRÔNICA}

\author{
CUIDADO DE ENFERMERÍA SOBRE VENTILACIÓN NO INVASIVA EN \\ PACIENTES CON ENFERMEDAD PULMONAR OBSTRUCTIVA CRÓNICA

\section{NURSING CARE ABOUT NON INVASIVE VENTILATION IN PATIENTS WITH CHRONIC OBSTRUCTIVE LUNG DISEASE}

\author{
Apresentação: Comunicação Oral \\ Francielle Rafaela dos Santos Silva ${ }^{1}$; André da Silva Santos²; Rayzza da Silva Ferreira ${ }^{3}$; Juliana Mendes \\ Correia ${ }^{4}$, Wilson Antonio da Silva ${ }^{5}$
}

DOI :https://doi.org/10.31692/2526-7701.IIICOINTERPDVS.0158

\begin{abstract}
RESUMO
A Doença Pulmonar Obstrutiva Crônica - DPOC é uma patologia que acomete as vias aéreas dos pacientes, caracterizado por uma obstrução no fluxo aéreo e uma das causas mais comuns para o desenvolvimento dessa doença é o tabagismo. O presente artigo tem por objetivo elencar os cuidados de Enfermagem acerca da Ventilação Não Invasiva - VNI e citar suas interfaces, em pacientes com DPOC. Trata-se de uma revisão integrativa com artigos presentes nas plataformas: SciELO, LILACS e BVS. As informações foram coletadas entre os anos de 2008 a 2021, em português e inglês. A assistência de Enfermagem frente a pacientes com Doença Pulmonar Obstrutiva Crônica - DPOC é de suma importância, pois a equipe de Enfermagem estará responsável junto com a equipe multidisciplinar por prestar cuidados seguros e eficazes, com o intuito de obter bons resultados ao tratamento do paciente. A assistência prestada para esses pacientes envolvidos em tratamento farmacológico dispõe-se em medicações inalatórias, frente a isso é notório a importância dos cuidados de Enfermagem para a administração correta com as interfaces utilizadas. A assistência de Enfermagem utilizada a pacientes com DPOC em uso de dispositivos inalatórios consistem em: realizar anamnese e exame físico, inspecionar a mucosa da narina antes e depois do procedimento, fornecer oxigenoterapia e manusear os dispositivos de forma segura. Além disso, torna-se indispensável obter uma comunicação efetiva entre os profissionais presentes no caso. Há diversos dispositivos utilizados que permeiam a VNI, como: máscara nasal, máscara orofacial e máscara facial total. Por fim, o profissional enfermeiro deve prestar cuidados de forma holística a pacientes em uso de VNI, bem como ter conhecimentos sobre as interfaces utilizadas nesse suporte ventilatório. Diante do exposto, resultará em um tratamento de sucesso e sem danos à saúde do paciente.
\end{abstract}

Palavras-chave: Ações de Enfermagem, Dispositivos Inalatórios e DPOC.

\footnotetext{
${ }^{1}$ Enfermagem, UniSãoMiguel, IIDV-PDVS, enfsilvafrancielle@gmail.com

${ }^{2}$ Enfermagem, UniSãoMiguel, IIDV-PDVS, andregpp5@gmail.com

${ }^{3}$ Nutrição, UniSãoMiguel, IIDV-PDVS, rayzzadasilvaferreira@ gmail.com

${ }^{4}$ Doutorado, UniSãoMiguel, IIDV-PDVS, mendescorre@ yahoo.com.br

${ }^{5}$ Licenciatura em Química, UFRPE, IIDV - PDVS, wilson.antonio98@ hotmail.com
} 


\section{RESUMEN}

Enfermedad Pulmonar Obstructiva Crónica - La EPOC es una patología que afecta las vías respiratorias de los pacientes, debido a una obstrucción en el flujo de aire y una de las causas más comunes para el desarrollo de esta enfermedad o el tabaquismo. Este artículo tiene como objetivo enumerar la atención de enfermería sobre ventilación no invasiva - VNI y citar sus interfaces en pacientes con EPOC. Se trata de una revisión integradora con artículos presentes en las plataformas: SciELO, LILACS y BVS. La información fue recolectada entre los años 2008 a 2021, en inglés e inglés. La atención de enfermería al paciente con Enfermedad Pulmonar Obstructiva Crónica - EPOC es de suma importancia, ya que el equipo de Enfermería debe ser responsable, junto con un equipo multidisciplinario, de brindar una atención segura y eficaz, con el fin de obtener buenos resultados en el tratamiento del paciente. La asistencia brindada a estos pacientes tratados en tratamiento farmacológico dispuesto en medicamentos inhalados, por lo que es notoria la importancia del cuidado de enfermería para la correcta administración con las interfaces utilizadas. La atención de enfermería utiliza a los pacientes con EPOC mediante dispositivos de inhalación, que consisten en: realizar anamnesis y exploración física, inspeccionar la mucosa de la fosa nasal antes y después del procedimiento, brindar oxigenoterapia y manipular los dispositivos de manera segura. Además, es fundamental obtener una comunicación eficaz entre los profesionales presentes en el caso. Se utilizan varios dispositivos que impregnan la VNI, como: mascarilla nasal, mascarilla orofacial y mascarilla facial completa. Finalmente, el enfermero profesional debe brindar atención integral a los pacientes que utilizan VNI, además de tener conocimiento sobre las interfaces utilizadas en este soporte ventilatorio. Dado lo anterior, resultará en un tratamiento exitoso sin dañar la salud del paciente.

Palabras-clave: Acciones de enfermería, inhaladores y EPOC.

\section{ABSTRACT}

Chronic Obstructive Pulmonary Disease - COPD is a pathology that affects the airways of patients, due to an obstruction in the airflow and one of the most common causes for the development of this disease or smoking. This article aims to list Nursing care about Non-Invasive Ventilation - NIV and cite its interfaces in patients with COPD. This is an integrative review with articles present in the platforms: SciELO, LILACS and VHL. The information was collected between the years 2008 to 2021, in english and english. Nursing care for patients with Chronic Obstructive Pulmonary Disease - COPD is of paramount importance, as the Nursing team must be responsible, together with a multidisciplinary team, for providing safe and effective care, in order to obtain good results in the patient's treatment. The assistance provided to these patients treated in pharmacological treatment arranged in inhaled medications, in view of this, the importance of nursing care for the correct administration with the interfaces used is notorious. Nursing care uses patients with COPD using inhalation devices, consisting of: conducting anamnesis and physical examination, inspecting the nostril mucosa before and after the procedure, providing oxygen therapy and handling the devices safely. In addition, it is essential to obtain effective communication between the professionals present in the case. There are several devices used that permeate the NIV, such as: nasal mask, orofacial mask and full face mask. Finally, the professional nurse must provide care holistically to patients using NIV, as well as having knowledge about the interfaces used in this ventilatory support. Given the above, it will result in a successful treatment and no harm to the patient's health.

Key words: Nursing Actions, inhalation devices and COPD.

\section{INTRODUÇÃO}

A doença pulmonar obstrutiva crônica (DPOC) é responsável por acometer as vias aéreas e como consequência acarretar na obstrução do fluxo aéreo, que pode se agravar gradualmente. Quanto aos fatores de predisposição, há como destaque o tabagismo, que resulta 
na destruição das paredes alveolares, tendo como consequência final um enfisema pulmonar e resultando no aprisionamento de ar nos pulmões (hiperinsuflação). O principal sintoma é caracterizado pela Dispnéia (dificuldade em respirar), consequentemente levando a uma incapacidade das atividades normais diárias, perdendo desta forma a qualidade de vida (SILVA et al., 2020).

A DPOC é reconhecida como um problema de saúde pública a nível mundial, pois a sua prevalência é bem característica em todos os países. Quanto à dados estáticos, estima-se que em 2030 a DPOC seja a quarta causa de mortalidade de pessoas no mundo. O público mais acometido por esta patologia são pessoas com mais de 40 anos e a maior prevalência no sexo masculino (SILVA et al., 2020).

Quanto ao tratamento da DPOC abrange um conjunto de ações voltadas para o paciente de forma holística, que pode ser classificada em assistência não farmacológica e farmacológica. No que se refere ao tratamento não-farmacológico, tem-se a prevenção de modo que ocorra a educação em saúde permanente, cessação do tabagismo e dentre outros. Já no tratamento farmacológico, agrega-se medicações inalatórias, principal tratamento utilizado frente a estes pacientes, com o intuito de prevenir crises exacerbadas. Este método de tratamento farmacológico é o mais utilizado, pois a sua forma permite uma ação de imediato, diminuindo os riscos de efeitos adversos (SILVA et al., 2020).

A ventilação não invasiva (VNI) tem a finalidade de promover alívio de sobrecarga da musculatura respiratória e demonstrou-se ser um forte procedimento e com resultados satisfatórios que tem por objetivo prevenir complicações futuras frente à pacientes com DPOC. Diante do exposto, após a prescrição médica, é de extrema importância a assistência de Enfermagem frente a administração correta com estes dispositivos (ALVARENGA; GARDENGHI, 2017).

Assim sendo, o presente estudo tem a finalidade de elencar os cuidados de Enfermagem acerca da VNI e citar suas interfaces, em pacientes com DPOC.

\section{FUNDAMENTAÇÃO TEÓRICA}

De acordo com Cabral e Gardenghi (2019) a DPOC resulta na limitação da distribuição do ar nas vias aéreas, tendo como causadores alguns gases tóxicos e partículas nocivas que resulta em dispneia, devido à diminuição da elasticidade dos pulmões, como também na resistência das paredes das vias aéreas.

De acordo com Alves et al., (2018) a VNI é um método não invasivo a via aérea e é 
utilizado através de interfaces como máscaras nasais ou faciais. Afirma também que, para utilização deste suporte ventilatório o paciente deve apresentar-se desperto e cooperativo, apresentando reflexos das vias aéreas superiores preservadas para que desta forma não acarrete risco ao pacientes como por exemplo broncoaspiração. Por fim, a VNI proporciona uma melhor oxigenação e prognóstico clínico, bem como diminui a necessidade de intubação do paciente com DPOC.

O uso de oxigênio através da VNI frente a pacientes com DPOC é necessário, pois o método objetiva diminuir toda sobrecarga fornecida na musculatura ventilatória, promovendo uma troca gasosa mais eficaz (CABRAL, GARDENGHI, 2019).

Alves et al., (2018) e Cabral, Gardenghi, (2019), afirmam que a VNI é um bom aliado para pacientes com DPOC, pois os resultados são satisfatórios, evita-se a intubação e possíveis complicações futuras a esses clientes.

Partindo desse olhar, os cuidados de Enfermagem devem atentar-se de forma contínua sobre a forma segura de manusear, conservar os dispositivos em geral disponíveis no setor. Além disso, os cuidados direcionados ao uso de dispositivos inalatórios desses pacientes tem como objetivo manter a perfusão e oxigenação adequada, com a finalidade de evitar hipóxia por meio da monitorização dos sinais vitais (ALVES et al., 2018).

Nessa dimensão, as interfaces que podem ser aplicadas a VNI consistem em máscara nasal, orofacial, facial total ou capacete. No entanto, para cada interface utilizada o volume interno referido será distribuído de forma diferente (REGO et al, 2012).

\section{METODOLOGIA}

O presente estudo trata-se de uma Revisão Integrativa baseada no modelo proposto por (ALVES et al., 2018). Esses autores desenvolveram um modelo de revisão integrativa fundamentada pelos seguintes critérios: I- Análise e leitura do título e resumo, II- Critérios de inclusão e exclusão, III- Inclusão dos resultados e discussões que contemplavam o assunto.

Foram pesquisados artigos científicos nas plataformas Scientific Electronic Library Online (SciELO), Literatura Latino-Americana e do Caribe em Ciências da Saúde (LILACS), Biblioteca Virtual em Saúde (BVS). As informações coletadas foram de artigos publicados entre os anos de 2008 a 2021, no idioma português e inglês, tendo como descritores: Ações de Enfermagem, dispositivos inalatórios e DPOC.

Para delimitação da pesquisa foram utilizados artigos que contemplam a temática a partir da análise do título e resumo. Tendo como critérios de exclusão, ano de publicação 
anterior a 2007, estudo de caso e tese.

A figura a seguir, tem como propósito informar a quantidade de artigos inclusos e exclusos de cada plataforma extraída. Diante do exposto, na plataforma SciELO observa-se que foram utilizados cinco artigos e zero excluído, na LILACS foi utilizado um artigo e um excluído, enquanto na BVS utilizou-se 17 e dois excluídos. Totalizando desta forma 23 artigos, porém apenas 20 utilizados e três exclusos.

Figura 1: Demonstração da quantidade de artigos inclusos e exclusos.
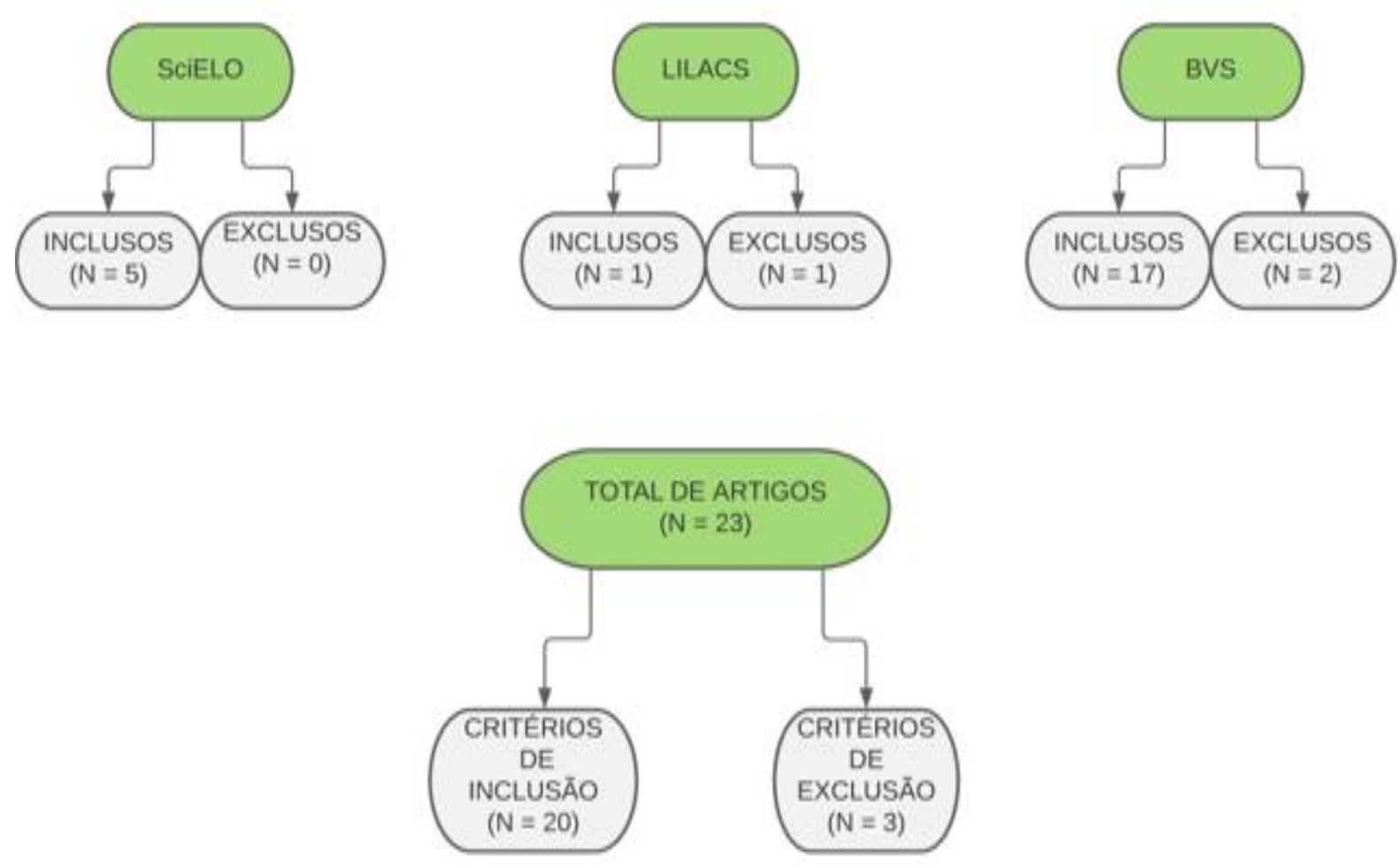

Fonte: Elaborado pelo autor (2021).

\section{RESULTADOS E DISCUSSÃO}

Para síntese da revisão foi utilizado o instrumento de coleta de dados de Ursi (2005) com modificações. No instrumento foram incluídos os nomes dos autores, títulos, objetivos e principais resultados. Após análise detalhada dos artigos encontrados percebeu-se que temas voltados para cuidados de Enfermagem acerca da VNI frente a pacientes com DPOC são frequentemente relatados nas publicações, sendo estes utilizados como categorias para a análise 
integrativa e discussão dos resultados.

A tabela abaixo contém um resumo dos principais artigos encontrados e que contribuíram para a elaboração do artigo. Destarte, são destacados os 20 artigos na tabela que foram divididos em tópicos de autores, títulos, objetivos e principais resultados.

Tabela 1: Resultados da busca de artigos relacionados a VNI em pacientes com DPOC e interfaces.

\begin{tabular}{|c|c|c|c|}
\hline Autores & Títulos & Objetivos & Principais Resultados \\
\hline AlVES et al., 2018 & $\begin{array}{l}\text { O papel do enfermeiro } \\
\text { na } \\
\text { oxigenoterapia:revisão } \\
\text { narrativa. }\end{array}$ & $\begin{array}{l}\text { Conhecer a produção } \\
\text { científica disponível } \\
\text { acerca da importância do } \\
\text { papel do enfermeiro e de } \\
\text { suas ações quanto à } \\
\text { oxigenoterapia. }\end{array}$ & $\begin{array}{l}\text { Os resultados obtidos a } \\
\text { partir do estudo } \\
\text { caracterizou-se sobre o } \\
\text { conhecimento do } \\
\text { Enfermeiro quanto à } \\
\text { assistência na } \\
\text { oxigenoterapia. }\end{array}$ \\
\hline CHIN et al., 2020 & $\begin{array}{c}\text { Aulas práticas na } \\
\text { implementação de } \\
\text { avaliações de fragilidade } \\
\text { para pacientes } \\
\text { hospitalizados com } \\
\text { DPOC. }\end{array}$ & $\begin{array}{l}\text { Avaliar a viabilidade de } \\
\text { implementação do CFS } \\
\text { entre pacientes } \\
\text { hospitalizados com } \\
\text { doença pulmonar } \\
\text { obstrutiva crônica } \\
\text { (DPOC). }\end{array}$ & $\begin{array}{l}\text { Foi possível identificar } \\
\text { que aproximadamente } \\
\text { vinte profissionais de } \\
\text { Enfermagem } \\
\text { completaram as } \\
\text { avaliações de fragilidade } \\
\text { para quarenta e seis de } \\
\text { cinquenta pacientes com } \\
\text { DPOC, entre os anos de } \\
2016 \text { e } 2017 .\end{array}$ \\
\hline $\begin{array}{c}\text { CABRAL; } \\
\text { GARDENGHI, } 2019\end{array}$ & $\begin{array}{c}\text { Análise sobre a } \\
\text { utilização e a eficácia da } \\
\text { ventilação não invasiva } \\
\text { em pacientes com } \\
\text { doença pulmonar } \\
\text { obstrutiva crônica. }\end{array}$ & $\begin{array}{l}\text { Analisar a utilização da } \\
\text { VNI e seus aspectos } \\
\text { quanto a sua eficácia no } \\
\text { tratamento de indivíduos } \\
\text { com DPOC. }\end{array}$ & $\begin{array}{c}\text { Constatou-se que a } \\
\text { Ventilação Não } \\
\text { Invasiva- VNI é eficaz e } \\
\text { acarreta na reversão } \\
\text { rápida do quadro de } \\
\text { DPOC. }\end{array}$ \\
\hline REGO et al., 2012 & $\begin{array}{c}\text { Avaliação do } \\
\text { desempenho de } \\
\text { diferentes interfaces para } \\
\text { ventilação não invasiva } \\
\text { em modelo mecânico } \\
\text { simulando paciente com } \\
\text { DPOC. }\end{array}$ & $\begin{array}{l}\text { Comparar o desempenho } \\
\text { de três interfaces para } \\
\text { VNI em termos de } \\
\text { sincronia paciente- } \\
\text { ventilador. }\end{array}$ & $\begin{array}{l}\text { Evidenciou-se que que } \\
\text { as máscaras que } \\
\text { apresentam menor } \\
\text { volume interno } \\
\text { obtiveram menor } \\
\text { vazamento e por este } \\
\text { motivo caracterizou pela } \\
\text { melhor escolha. }\end{array}$ \\
\hline $\begin{array}{l}\text { PADILHA; OLIVEIRA; } \\
\text { CAMPOS, } 2010\end{array}$ & $\begin{array}{l}\text { Revisão integrativa da } \\
\text { literatura sobre gestão do } \\
\text { regime terapêutico em } \\
\text { pacientes com doença } \\
\text { pulmonar obstrutiva } \\
\text { crônica. }\end{array}$ & $\begin{array}{l}\text { Identificar qual a melhor } \\
\text { estratégia de intervenção } \\
\text { de enfermagem na } \\
\text { promoção da gestão do } \\
\text { regime terapêutico em } \\
\text { pacientes com Doença } \\
\text { Pulmonar Obstrutiva } \\
\text { Crônica no ambiente } \\
\text { hospitalar. }\end{array}$ & $\begin{array}{l}\text { Pode-se observar que os } \\
\text { estudos utilizam diversos } \\
\text { indicadores de } \\
\text { efetividade para } \\
\text { avaliarem as estratégias } \\
\text { utilizadas nos regimes } \\
\text { terapêuticos. Sendo } \\
\text { assim, não é possível } \\
\text { identificar o melhor } \\
\text { regime a ser utilizado. }\end{array}$ \\
\hline
\end{tabular}




\begin{tabular}{|c|c|c|c|}
\hline SILVA et al., 2020 & $\begin{array}{l}\text { Avaliação do manuseio } \\
\text { de dispositivos } \\
\text { inalatórios em pacientes } \\
\text { com doença pulmonar } \\
\text { obstrutiva crônica em } \\
\text { um hospital terciário. }\end{array}$ & $\begin{array}{l}\text { Avaliar a técnica de uso } \\
\text { dos dispositivos } \\
\text { inalatórios em pacientes } \\
\text { portadores de DPOC, } \\
\text { atendidos no ambulatório } \\
\text { de pneumologia de um } \\
\text { Hospital Terciário na } \\
\text { cidade do Recife-PE. }\end{array}$ & $\begin{array}{l}\text { Foi identificado no } \\
\text { estudo que os } \\
\text { determinantes sociais } \\
\text { como: baixa escolaridade } \\
\text { e renda salarial baixa } \\
\text { foram os condicionantes } \\
\text { responsáveis pela má } \\
\text { adesão a dispositivos } \\
\text { inalatórios. }\end{array}$ \\
\hline $\begin{array}{c}\text { BASTOS; } \\
\text { VIEIRA; } \\
\text { LIMA, 2019 }\end{array}$ & $\begin{array}{c}\text { Gestão do regime } \\
\text { farmacológico em } \\
\text { doentes com doença } \\
\text { pulmonar obstrutiva } \\
\text { crônica }\end{array}$ & $\begin{array}{c}\text { Estudar a adesão e a } \\
\text { competência percebida } \\
\text { das pessoas para } \\
\text { cumprirem o regime de } \\
\text { tratamento da DPOC e } \\
\text { avaliar o seu } \\
\text { conhecimento sobre o } \\
\text { uso de inaladores. }\end{array}$ & $\begin{array}{c}\text { O regime terapêutico } \\
\text { apresentou uma boa } \\
\text { adesão para a DPOC, } \\
\text { mas quanto ao uso de } \\
\text { dispositivos inalatórios } \\
\text { os usuários apresentaram } \\
\text { pouco conhecimento } \\
\text { para o uso correto e } \\
\text { consequentemente } \\
\text { refletindo no } \\
\text { agravamento do caso } \\
\text { clínico. }\end{array}$ \\
\hline WANG et al., 2020 & $\begin{array}{l}\text { O efeito de um programa } \\
\text { de autogestão liderado } \\
\text { por enfermeiras nos } \\
\text { resultados de pacientes } \\
\text { com doença pulmonar } \\
\text { obstrutiva crônica }\end{array}$ & $\begin{array}{l}\text { Para examinar a eficácia } \\
\text { de um programa de } \\
\text { autogestão liderado por } \\
\text { enfermeiros em } \\
\text { resultados de pacientes } \\
\text { com doença pulmonar } \\
\text { obstrutiva crônica } \\
\text { (DPOC). }\end{array}$ & $\begin{array}{l}\text { Comparado ao grupo de } \\
\text { controle, os } \\
\text { colaboradores do grupo } \\
\text { de intervenção } \\
\text { demonstraram uma } \\
\text { menor hospitalização } \\
\text { relacionada a DPOC. }\end{array}$ \\
\hline TASOTA et al., 2021 & $\begin{array}{l}\text { Cânula nasal ou oxigênio } \\
\text { de alto } \\
\text { fluxo para pacientes com } \\
\text { DPOC em } \\
\text { dificuldade respiratória } \\
\text { aguda }\end{array}$ & $\begin{array}{l}\text { Abordar um equívoco } \\
\text { comum da enfermagem } \\
\text { em relação ao uso da } \\
\text { administração de } \\
\text { oxigênio de alto fluxo } \\
\text { por meio de máscaras. }\end{array}$ & $\begin{array}{l}\text { Foi constatado no estudo } \\
\text { que os Enfermeiros } \\
\text { sendo eles experientes } \\
\text { ou não optaram por um } \\
\text { tratamento de } \\
\text { oxigenoterapia em baixo } \\
\text { fluxo em vez de alto } \\
\text { fluxo à pacientes com } \\
\text { DPOC. }\end{array}$ \\
\hline STRANG et al., 2019 & $\begin{array}{l}\text { Apoio a cuidadores } \\
\text { informais de pacientes } \\
\text { com doença pulmonar } \\
\text { obstrutiva crônica grave: } \\
\text { um estudo qualitativo } \\
\text { das experiências de } \\
\text { cuidadores e } \\
\text { profissionais em } \\
\text { hospitais suecos. }\end{array}$ & $\begin{array}{l}\text { Descrever as percepções } \\
\text { sobre o apoio à saúde } \\
\text { para cuidadores } \\
\text { informais, tanto na } \\
\text { perspectiva do cuidador } \\
\text { familiar quanto da } \\
\text { equipe. }\end{array}$ & $\begin{array}{c}\text { A partir do estudo } \\
\text { evidenciou-se que os } \\
\text { cuidadores informais ou } \\
\text { quanto a equipe } \\
\text { apresentavam } \\
\text { dificuldade em fornecer } \\
\text { apoio adequado à } \\
\text { pacientes com DPOC e } \\
\text { suas famílias, desta } \\
\text { forma os cuidadores } \\
\text { necessitavam de apoio } \\
\text { emocional, prático. Já a } \\
\text { equipe não sabia ao certo } \\
\text { os seus deveres em } \\
\text { relação a família. }\end{array}$ \\
\hline
\end{tabular}




\begin{tabular}{|c|c|c|c|}
\hline ZWAKMAN et al., 2019 & $\begin{array}{c}\text { Pacientes perspectivas } \\
\text { sobre a intervenção } \\
\text { DPOC- } \\
\text { GRIP, um novo cuidado } \\
\text { de enfermagem } \\
\text { intervenção para DPOC. }\end{array}$ & $\begin{array}{c}\text { Explorar pacientes } \\
\text { experientes sobre a } \\
\text { intervenção DPOC- } \\
\text { GRIP. }\end{array}$ & $\begin{array}{l}\text { Com a intervenção do } \\
\text { DPOC-GRIP foi possível } \\
\text { observar nos pacientes a } \\
\text { boa aceitação, pois os } \\
\text { mesmos relataram um } \\
\text { melhor acolhimento. }\end{array}$ \\
\hline $\begin{array}{l}\text { LELLOUCHE et al., } \\
2016\end{array}$ & $\begin{array}{l}\text { Titulação de oxigênio } \\
\text { automatizada e desmame } \\
\text { livre do O2 em pacientes } \\
\text { com exacerbação aguda } \\
\text { de DPOC: um ensaio } \\
\text { piloto randomizado. }\end{array}$ & $\begin{array}{l}\text { Avaliar a viabilidade do } \\
\text { uso do livre } \mathrm{O} 2 \mathrm{em} \\
\text { pacientes hospitalizados } \\
\text { na enfermaria } \\
\text { respiratória por uma } \\
\text { exacerbação aguda da } \\
\text { DPOC. }\end{array}$ & $\begin{array}{l}\text { Enfermeiros e médicos } \\
\text { assistentes avaliaram que } \\
\text { os dois sistemas de } \\
\text { administração eram } \\
\text { igualmente apropriados. }\end{array}$ \\
\hline MACCARI et al., 2015 & $\begin{array}{l}\text { Terapia inalatória em } \\
\text { ventilação mecânica. }\end{array}$ & $\begin{array}{l}\text { Esse estudo evidencia a } \\
\text { heterogeneidade na } \\
\text { prescrição de drogas } \\
\text { inalatórias, } \\
\text { demonstrando a pobre } \\
\text { aplicação do } \\
\text { conhecimento científico } \\
\text { atual. }\end{array}$ & $\begin{array}{l}\text { Foi possível identificar } \\
\text { que muitos fatores } \\
\text { podem interferir de } \\
\text { acordo com os } \\
\text { medicamentos } \\
\text { broncodilatadores, por } \\
\text { exemplo, modo de } \\
\text { ventilatório e tamanho } \\
\text { do tubo. }\end{array}$ \\
\hline BLAHA et al., 2018 & $\begin{array}{l}\text { Gestão de hospitalização } \\
\text { de pacientes com DPOC. }\end{array}$ & $\begin{array}{l}\text { Este projeto de melhoria } \\
\text { incorporou intervenções } \\
\text { de enfermagem com } \\
\text { base na teoria de } \\
\text { transições e diretrizes } \\
\text { publicadas do American } \\
\text { College of Chest } \\
\text { Physicians e da } \\
\text { Canadian Thoracic } \\
\text { Society no desenho de } \\
\text { uma iniciativa de } \\
\text { redução da readmissão } \\
\text { da DPOC. }\end{array}$ & $\begin{array}{c}\text { Identificou-se que } \\
\text { existem algumas } \\
\text { lacunas, como a falta } \\
\text { ocasional de } \\
\text { acompanhamento } \\
\text { especializado, } \\
\text { prescrições } \\
\text { inconsistentes, } \\
\text { cumprimento da } \\
\text { medicação programada } \\
\text { não realizada, como } \\
\text { também o não } \\
\text { acompanhamento pós- } \\
\text { alta são responsáveis } \\
\text { pela readmissão da } \\
\text { DPOC. }\end{array}$ \\
\hline SOUZA et al., 2009 & $\begin{array}{c}\text { Técnica e compreensão } \\
\text { do uso dos dispositivos } \\
\text { inalatórios em pacientes } \\
\text { com asma ou DPOC }\end{array}$ & $\begin{array}{l}\text { Avaliar a compreensão e } \\
\text { a técnica de uso dos } \\
\text { dispositivos inalatórios } \\
\text { prescritos de pacientes } \\
\text { com asma ou DPOC } \\
\text { atendidos em um } \\
\text { hospital-escola terciário. }\end{array}$ & $\begin{array}{c}\text { Foram avaliados } 120 \\
\text { voluntários: } 60 \text { com } \\
\text { asma e } 60 \text { com DPOC. } \\
\text { Ao usar aerossóis } \\
\text { quantitativos }(\mathrm{p}= \\
0,0023), \text { Pulvinal }(\mathrm{p}= \\
0,0065) \text { e Aerolizer }(\mathrm{p} \\
=0,012), \text { o grupo DPOC } \\
\text { apresentou uma taxa de } \\
\text { erro significativamente } \\
\text { maior do que o grupo } \\
\text { asma. }\end{array}$ \\
\hline
\end{tabular}




\begin{tabular}{|c|c|c|c|}
\hline $\begin{array}{c}\text { DE VASCONCELOS et } \\
\text { al., } 2015\end{array}$ & $\begin{array}{l}\text { Prevalência do uso } \\
\text { inadequado de } \\
\text { dispositivos inalatórios } \\
\text { por pacientes com asma } \\
\text { e/ou DPOC atendidos } \\
\text { em ambulatório } \\
\text { especializado. }\end{array}$ & $\begin{array}{c}\text { Avalia o uso de } \\
\text { dispositivos inalatórios } \\
\text { por pacientes no HUAC- } \\
\text { UFCG. Foi realizado um } \\
\text { estudo transversal com } \\
91 \text { usuários com asma } \\
\text { e/ou DPOC, em uso de } \\
\text { medicações através de } \\
\text { dispositivos inalatórios } \\
\text { do tipo inaladores } \\
\text { pressurizados (IP) e/ou } \\
\text { inaladores de pó seco } \\
\text { (IPS). }\end{array}$ & $\begin{array}{l}\text { A maioria dos pacientes } \\
\text { usa o dispositivo de } \\
\text { forma incorreta ( } 93,3 \% \\
\text { para IP e } 85,2 \% \text { para } \\
\text { IPS). O erro mais } \\
\text { comum nos dois } \\
\text { dispositivos é não } \\
\text { realizar a expiração } \\
\text { normal antes do uso (IP } \\
\text { é } 92,9 \%, \text { IPS é } 90,7 \%)\end{array}$ \\
\hline MACHADO et al., 2015 & $\begin{array}{l}\text { Prevalência e preditores } \\
\text { do uso incorreto dos } \\
\text { dispositivos inalatórios } \\
\text { em pacientes portadores } \\
\text { de doença pulmonar } \\
\text { obstrutiva crônica }\end{array}$ & $\begin{array}{c}\text { Inferir a prevalência e } \\
\text { preditores do uso } \\
\text { incorreto dos } \\
\text { dispositivos inalatórios } \\
\text { em pacientes } \\
\text { ambulatoriais portadores } \\
\text { de doença pulmonar } \\
\text { obstrutiva crônica } \\
\text { (DPOC). }\end{array}$ & $\begin{array}{c}\text { A prevalência de } \\
\text { indivíduos que fizeram } \\
\text { mau uso do dispositivo } \\
\text { inalatório foi de } 96 \% \text { ( } 24 \\
\text { pessoas). Observou-se } \\
\text { que o erro médio entre } \\
\text { os grupos com um } \\
\text { aparelho foi } \\
\text { significativamente } \\
\text { menor do que o grupo } \\
\text { com três aparelhos (p = } \\
0,04) .\end{array}$ \\
\hline OLIVEIRA et al., 2014 & $\begin{array}{l}\text { Avaliação da técnica de } \\
\text { utilização de dispositivos } \\
\text { inalatórios no tratamento } \\
\text { de doenças respiratórias } \\
\text { no sul do Brasil: estudo } \\
\text { de base populacional. }\end{array}$ & $\begin{array}{c}\text { Conhecer os erros na } \\
\text { técnica de uso de } \\
\text { dispositivos inalatórios } \\
\text { empregada por pacientes } \\
\text { com doenças } \\
\text { respiratórias no sul do } \\
\text { Brasil e o perfil daqueles } \\
\text { que possuem } \\
\text { dificuldades em realizá- } \\
\text { la. }\end{array}$ & $\begin{array}{c}\text { Os principais erros } \\
\text { identificados quanto ao } \\
\text { uso de IPrs e IP foram } \\
\text { não expirar antes da } \\
\text { inalação e não fazer uma } \\
\text { pausa inspiratória após a } \\
\text { inalação. }\end{array}$ \\
\hline $\begin{array}{l}\text { SILVESTRI; PEREIRA; } \\
\text { RODRIGUES, } 2008\end{array}$ & $\begin{array}{c}\text { Comparação da variação } \\
\text { de resposta ao } \\
\text { broncodilatador através } \\
\text { da espirometria em } \\
\text { portadores de asma ou } \\
\text { doença pulmonar } \\
\text { obstrutiva crônica }\end{array}$ & $\begin{array}{l}\text { O diagnóstico diferencial } \\
\text { entre asma e doença } \\
\text { pulmonar obstrutiva } \\
\text { crônica (DPOC) através } \\
\text { da resposta aos } \\
\text { broncodilatadores } \\
\text { inalatórios na } \\
\text { espirometria ainda é } \\
\text { controverso. O objetivo } \\
\text { deste estudo foi detectar } \\
\text { quais variáveis } \\
\text { espirométricas melhor } \\
\text { diferenciam asma de } \\
\text { DPOC. }\end{array}$ & $\begin{array}{c}\text { O volume expiratório } \\
\text { forçado (VEF1) um } \\
\text { segundo antes do } \\
\text { broncodilatador ser } \\
\text { usado nos dois grupos } \\
\text { era o mesmo (VEF1 = } \\
51 \% \text { ), mas os pacientes } \\
\text { com DPOC eram mais } \\
\text { velhos (66 } \pm \text { anos vs. } \\
59 \pm 11 \text { anos, p <0,001), } \\
\text { e a maioria deles era do } \\
\text { sexo masculino (73\% vs. } \\
27 \%, \text { p <0,001). A } \\
\text { variação absoluta } \\
\text { mediana de VEF1 após } \\
\text { broncodilatador foi de } \\
0,25 \mathrm{~L} \text { (variação, -0,09 a } \\
1,13 \mathrm{~L} \text { ) em pacientes } \\
\text { com asma e } 0,09 \mathrm{~L} \\
\text { (variação de }-0,1 \text { a } 0,73 \\
\mathrm{~L} \text { ) em pacientes com } \\
\text { DPOC (p <0,001). }\end{array}$ \\
\hline
\end{tabular}




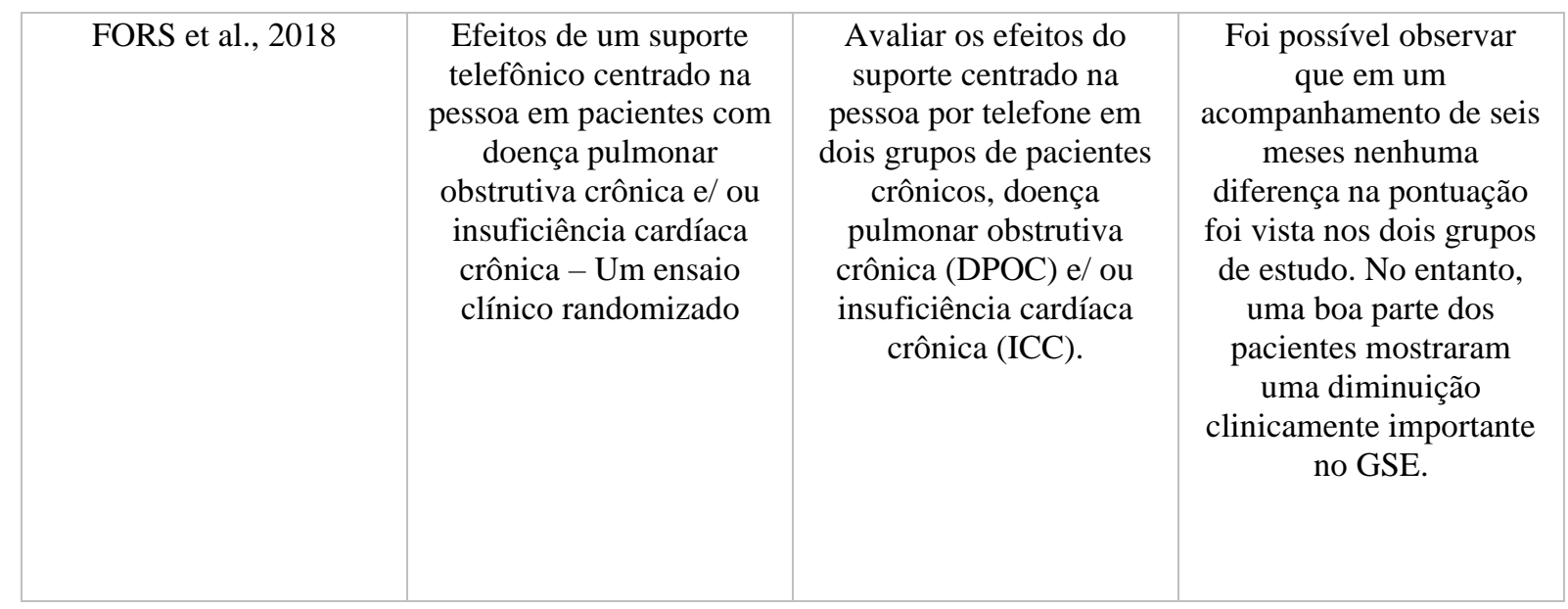

Fonte: Elaborado pelo autor (2021).

Pode-se constatar que o enfermeiro na aplicabilidade de conhecimento do uso de oxigenoterapia frente à pacientes com DPOC pode fortalecer quanto aos seus cuidados. Além disso, a equipe de Enfermagem pode explorar na prática hospitalar a implementação de avaliações de fragilidade, variando o grau entre frágeis ou moderadamente frágeis, pois desta forma avaliou-se o nível de comprometimento das atividades diárias frente à pacientes com exacerbação da DPOC (SILVA et al., 2020).

Quanto ao uso da ventilação não invasiva- VNI, trata-se de um tratamento mais eficaz, sobretudo em pacientes que estão com sobrecarga e stress sofrido na musculatura respiratória, atuando desta forma para reversão do quadro (SILVA et al., 2020).

No que se refere ao uso inadequado de dispositivos inalatórios foi possível averiguar alguns determinantes negativos para um déficit a este tratamento, desta forma, a renda salarial baixa, como também o baixo nível de escolaridade são determinantes para a má adesão à técnica inalatória. Ademais, nos estudos foram utilizados testes para verificar as diferenças da DPOC nos grupos apresentados, quanto a gênero, idade, estilo de vida, tabagistas e entre outros grupos. Por fim, o uso de oxigênio de alto fluxo frente à pacientes com DPOC é inapropriado, desta forma oxigenoterapia de baixo fluxo e alta pressão é o mais adequado para o tratamento (SILVA et al., 2020).

Torna-se comum a presença da VNI em enfermarias, unidade de terapia intensiva- UTI e em pronto-socorros que atende pacientes apresentando crises exacerbadas de DPOC. A VNI é a mais recomendada, portanto traz efeitos significativos ao paciente como também trata insuficiência respiratória, quadro de acidose respiratória $\mathrm{pH}$ 7,35, dispneia moderada ou grave e consequentemente melhora no desempenho das atividades de vida diária (REGO et al., 2012).

Vale destacar que o uso da VNI deve ser em pacientes que apresentam estabilidade 
hemodinâmica, como também está com as vias aéreas permeáveis pois desta forma precaver contra a broncoaspiração (ALVES et al., 2018).

A indicação e a orientação quanto ao uso de dispositivos inalatórios é restrita para os médicos. No entanto, a assistência prestada a pacientes com DPOC é de responsabilidade multidisciplinar. A partir do exposto, a equipe de Enfermagem é de suma importância e está diretamente ligada na assistência (SILVA et al., 2020).

Logo, a equipe de Enfermagem estará responsável por prestar assistência segura de modo contínuo, a partir do manuseio, conservação e manutenção das interfaces como troca de filtro e umidificador dentro do prazo estabelecido do protocolo da Comissão de Controle de Infecção Hospitalar - CCIH de acordo com cada instituição. Além disso, deve-se observar rigorosamente a cavidade oral do paciente (ALVES et al., 2018).

É da competência do Enfermeiro na prática realizar anamnese e exame físico do tórax. Além disso, o intuito da equipe de Enfermagem é fornecer uma oxigenação adequada e segura, manter a sua perfusão e diminuir ou evitar crises exacerbadas desenvolvidas pela DPOC, bem como a inspeção da mucosa da narina antes e depois do procedimento, observar quanto a fixação e proteção da pele (ocorre com a oferta de água destilada estéril no umidificador), monitorar os sinais vitais e a manter a comunicação efetiva entre os profissionais envolvidos (ALVES et al., 2018).

Existem diversos dispositivos que permitem aplicar a VNI, podendo ser: máscara nasal (que tem a finalidade de cobrir o nariz, mas não a boca), máscara orofacial (cobre o nariz, como também a boca), máscara facial total (tem o intuito de cobrir nariz, boca e olhos) e capote (que recobre toda a região da cabeça e parte do pescoço). A máscara nasal é recomendado a pacientes apresentando insuficiência respiratória crônica, pois permite uma área mais livre e portanto a fala e a tosse estarão acessíveis, já as máscaras orofaciais são indicadas à pacientes com insuficiência respiratória aguda que permite um maior volume a ser expirado quando é coberto a região da boca e por fim a máscara facial total ou o capote são preferíveis em situações de intolerância à máscara orofacial, devido a lesão de mucosa ou até mesmo por serem mais confortáveis. Vale ressaltar que a escolha da máscara interfere no volume interno e pode influenciar diretamente no resultado do tratamento do paciente (REGO et al., 2012).

\section{CONCLUSÕES}

Diante do exposto, é notório a importância da equipe de Enfermagem frente à pacientes com DPOC, visto que a assistência a ser prestada deve ser de forma holística, baseada em 
evidências técnicas e científicas. Ressalta-se a importância do profissional Enfermeiro aprofundar seus conhecimentos sobre o aparelho respiratório, bem como estar ciente sobre as assistências prestadas que lhe cabe com o intuito de evitar danos à saúde do paciente, consequentemente obter sucesso no tratamento (ALVES et al., 2018).

Deste modo, a educação permanente, assim como a implementação rigorosa de protocolos baseados em evidências científicas e a padronização da assistência de Enfermagem resultará em qualidade de vida ao paciente hospitalizado (ALVES et al., 2018).

Ademais, é indispensável os cuidados da Enfermagem direcionado ao manuseio, conservação de dispositivos inalatórios em geral, especificamente a VNI, bem como os cuidados direcionados diretamente a pacientes em uso desse suporte ventilatório, pois desta forma os resultados a serem obtidos a partir de uma assistência holística do cliente e a escolha da interface a ser utilizada, tornam-se imprescindíveis para promover uma assistência de qualidade e obter resultados satisfatórios no tratamento (ALVES et al., 2018).

Por fim, é notório a limitação de artigos recentes relacionados à assistência da Enfermagem acerca de dispositivos inalatórios a pacientes com DPOC. Posto isto, este artigo objetiva contribuir para uma assistência de Enfermagem com qualidade.

\section{REFERÊNCIAS}

ALVES, J. C. F. et al. O papel do enfermeiro na oxigenoterapia: revisão narrativa da literatura. Journal of Health \& Biological Sciences, v. 6, n. 2, p. 176-181, 2018.

BASTOS, C; VIEIRA, S; LIMA, L. Gestão do regime farmacológico em doentes com doença pulmonar obstrutiva crônica. Revista de Enfermagem Referência, v. 4, n. 20, p. 57-65, 2019.

BLAHA, D. et al. Post hospitalization management of patients with COPD. Nursing management, v. 49, n. 1, p. 14-21, 2018.

CABRAL, D. Análise sobre a utilização e a eficácia da ventilação não invasiva em pacientes com doença pulmonar obstrutiva crônica. 2019.10 f. Trabalho de Pós graduação em fisioterapia intensiva, Faculdade CEAFI, Goiânia.

CHIN, M. et al. Practical lessons in implementing frailty assessments for hospitalised patients with COPD. BMJ open quality, v. 9, n. 1, p. e000782, 2020.

DE VASCONCELOS, I. M. M. et al. Prevalência do uso inadequado de dispositivos inalatórios por pacientes com asma e/ou dpoc atendidos em ambulatório especializado. Revista Saúde \& Ciência Online, v. 4, n. 2, p. 6-18, 2015.

FORS, A. et al. Effects of a person-centred telephone-support in patients with chronic 
obstructive pulmonary disease and/or chronic heart failure-A randomized controlled trial. PLoS One, v. 13, n. 8, p. e0203031, 2018.

LELLOUCHE, F. et al. Automated oxygen titration and weaning with FreeO2 in patients with acute exacerbation of COPD: a pilot randomized trial. International journal of chronic obstructive pulmonary disease, mv. 11, p. 1983, 2016.

MACCARI, J. G. et al. Terapia inalatória em ventilação mecânica. Jornal Brasileiro de Pneumologia, v. 41, p. 467-472, 2015.

MACHADO, G. C. et al. Prevalência e preditores do uso incorreto dos dispositivos inalatórios em pacientes portadores de Doença Pulmonar Obstrutiva Crônica Prevalence and predictors of incorrect use of inhaler devices in patients with COPD. Revista Educação em Saúde, v. 3, n. 2, 2015 .

OLIVEIRA, P. D. et al. Avaliação da técnica de utilização de dispositivos inalatórios no tratamento de doenças respiratórias no sul do Brasil: estudo de base populacional. Jornal Brasileiro de Pneumologia, v. 40, p. 513-520, 2014.

PADILHA, J. M. S. C; OLIVEIRA, M. F. S; CAMPOS, M. J. A. Revisão integrativa da literatura sobre gestão do regime terapêutico em pacientes com doença pulmonar obstrutiva crônica. Revista da Escola de Enfermagem da USP, v. 44, p. 1129-1134, 2010.

REGO, F. M. P. et al. Avaliação do desempenho de diferentes interfaces para ventilação não invasiva em modelo mecânico simulando paciente com DPOC. Revista de Medicina, v. 91, n. 2, p. 60-68, 2012.

SILVA, L. F. A. et al. Avaliação do manuseio de dispositivos inalatórios em pacientes com doença pulmonar obstrutiva crônica em um hospital terciário. Nursing (São Paulo), p. 3537$3542,2020$.

SILVESTRI, I. C; PEREIRA, C. A. C; RODRIGUES, S. C. S. Comparação da variação de resposta ao broncodilatador através da espirometria em portadores de asma ou doença pulmonar obstrutiva crônica. Jornal Brasileiro de Pneumologia, v. 34, p. 675-682, 2008.

SOUZA, M. L. M. et al. Técnica e compreensão do uso dos dispositivos inalatórios em pacientes com asma ou DPOC. Jornal Brasileiro de Pneumologia, v. 35, p. 824-831, 2009.

STRANG, S. et al. Support to informal caregivers of patients with severe chronic obstructive pulmonary disease: a qualitative study of caregivers' and professionals' experiences in Swedish hospitals. BMJ open, v. 9, n. 8, p. e028720, 2019.

TASOTA, F. J. et al. Nasal cannula or high-flow oxygen for patients with COPD in acute respiratory distress? Nursing 2021, v. 51, n. 5, p. 52-57, 2021.

WANG, L. H. et al. The effect of a nurse-led self-management program on outcomes of patients with chronic obstructive pulmonary disease. The clinical respiratory journal, v. 14, n. 2, p. 148-157, 2020.

ZWAKMAN, M. et al. Patients' perspectives on the COPD-GRIP intervention, a new nursing 
care intervention for COPD. BMC family practice, v. 20, n. 1, p. 1-8, 2019.

SOUZA, Maria Luiza de Moraes et al. Técnica e compreensão do uso dos dispositivos inalatórios em pacientes com asma ou DPOC. Jornal Brasileiro de Pneumologia, v. 35, p. 824-831, 2019. 